\title{
Effectiveness and Parental Perception of Silver Diamine Fluoride toward Treatment of Dental Caries in Primary Teeth
}

\author{
Upasana Shrivastava ${ }^{1}$, Khushboo Barjatya ${ }^{2}$, Bharath B AK ${ }^{3}$, Ankur Vatsal ${ }^{4}$, Rahul Shrivastava ${ }^{5}$, Abhilasha Manker $^{6}$, \\ Binti R Chand ${ }^{7}$, Preene Juneja ${ }^{8}$
}

\begin{abstract}
Aim: Silver diamine fluoride (SDF) is taken into account as one among the effective measures for caries arrest and prevention in youngsters, due to the fact it proffers marginally invasive but cost-effective alternative to time-commemorated restorative caries control. The study was carried out to appraise the effectiveness of SDF (38\%) in arresting active carious lesions and to gauge parental perception toward the treatment of cavities with SDF in the primary teeth.

Materials and methods: A total of 36 children in the age range 2-10 years, having active caries lésions, based on ICDAS criteria, in primary teeth were selected following the baseline examination. After four dropouts and refusal by two patients, 30 patients were treated with 1-2 topical applications of SDF (38\%). Lesions were reassessed at 1 week and 3 months taking into account to assess the color and amend inconsistency (soft/hard). Parents have been mentioned regarding features of pain or infection and have been surveyed with regard to subjective feelings regarding SDF.

Results: Quantitative variables have been subjected to the Chi-square test. All the 30 participants within the present study at baseline were reported to possess active caries lesion. About $80 \%$ of carious lesions were found to be arrested at first recall and $93.3 \%$ after 3 months. No occurrence of pain or infection was documented. Parental perception for simple application, taste, and esthetics became statistically significant. Conclusion: Study outcomes endorse SDF to be effective in preventing active carious lesions in deciduous dentition in youngsters, and treatment outcomes were affirmative by the parents.

Clinical significance: SDF has been a possible management option for a noninvasive remedy to inhibit active carious lesions in deciduous dentition without causing any psychological trauma to children.

Keywords: Dental caries, Esthetics, Parental perception, Primary teeth, Silver diamine fluoride.

International Journal of Clinical Pediatric Dentistry (2021): 10.5005/jp-journals-10005-1810
\end{abstract}

\section{INTRODUCTION}

Dental caries is the widely accepted collective non-communicable disease and affects children of various socioeconomic conditions throughout their lifetime, causing pain, distress, and sometimes disfigurement. ${ }^{1}$ It is multifactorial with tooth, food substrate, and microorganism, and time is a key factor. Other factors such as age, ethnicity, diet pattern, and socioeconomic status are variables in different regions, and individuals have also been recognized as the other contributing factors. ${ }^{2}$

The conventional management of decayed teeth involves the removal of infected and demineralized dental tissue and subsequent restoration, which may require the highly developed skill of the dentist as well as extensive instrumentation and costly restorative materials. Further, fear of dental treatment is quite common in children owing to their inadequate adaptive capacity. Hence, managing a carious tooth in such children can be more challenging for the dentist. In these circumstances, caries progression inhibition is proposed for managing active carious lesions. $^{3}$

Silver diamine fluoride (SDF) in different concentrations is a successful treatment for arresting caries and also prevents the occurrence of new carious lesions. It is considered a noninvasive technique that is cheap, swift, and simple to use. It has great potential, especially for early childhood caries, fearful adults, special needs children and adults, patients with salivary dysfunction, and patients with treatment challenged by behavioral or medical issues.

\begin{abstract}
1-3,6-8 Department of Pediatric and Preventive Dentistry, Sri Aurobindo Institute of Medical Sciences, Indore, Madhya Pradesh, India

${ }^{4}$ Department of Public Health Dentistry, Sri Aurobindo Institute of Medical Sciences, Indore, Madhya Pradesh, India

${ }^{5}$ Department of OMR, Government Dental College, Raipur, Chhattisgarh, India

Corresponding Author: Upasana Shrivastava, Department of Pediatric and Preventive Dentistry, Sri Aurobindo Institute of Medical Sciences, Indore, Madhya Pradesh, India, Phone: +91 8989120218, e-mail: dr.upasana123@gmail.com

How to cite this article: Shrivastava U, Barjatya $\mathrm{K}, \mathrm{AK} B \mathrm{~B}$, et al. Effectiveness and Parental Perception of Silver Diamine Fluoride toward Treatment of Dental Caries in Primary Teeth. Int J Clin Pediatr Dent 2021;14(6):790-794.
\end{abstract}

Source of support: : Nil

Conflict of interest: : None

Also, the SDF application does not require complex training of the dental or health professionals. ${ }^{4}$

SDF claims to interrupt the caries evolution and concurrently avert the development of a new lesion. SDF application is reported to be minimally invasive, easy, and also being cost-effective. ${ }^{5}$ An application of SDF causes dark stains on enamel and dentin, and it does raise esthetic concerns for children and parents as well. However, different parents have

() The Author(s). 2021 Open Access This article is distributed under the terms of the Creative Commons Attribution 4.0 International License (https://creativecommons. org/licenses/by-nc/4.0/), which permits unrestricted use, distribution, and non-commercial reproduction in any medium, provided you give appropriate credit to the original author(s) and the source, provide a link to the Creative Commons license, and indicate if changes were made. The Creative Commons Public Domain Dedication waiver (http://creativecommons.org/publicdomain/zero/1.0/) applies to the data made available in this article, unless otherwise stated. 
different perceptions toward using SDF cogitating its merits and demerits. ${ }^{5}$ Although parental perception stands to be evaluated in different Western countries, to the best of our knowledge, no such study is being carried out in the central Indian population.

The present study was conducted to assess the effectiveness of SDF (38\%) in preventing active dental caries in primary teeth and the parental perception toward the treatment of dental caries with SDF.

\section{Materials and Methods}

The present short-term clinical study was initiated following clearance from the institutional ethical and research board committee. Parents and patients willing to take part in the study were enrolled. Children in the age range $2-10$ years with at least one active untreated carious lesion falling under the category laid by ICDAS 3 and 4, that is, non-cavitated lesions, and ICDAS categories 5 and 6 , that is, active (soft) cavitated carious lesions in primary dentition, stretching out into dentin were included. ${ }^{6}$

Children having pain triggered unprompted or elicited from caries, tooth mobility, or signs of pulpal involvement; severe systemic health conditions that would not permit execution in the clinic; known allergies or sensitivities to SDF; hereditary developmental defects; and incapacity to cooperate for SDF handling or return for follow-up visits at 3 weeks and 3 months were debarred from the study.

Baseline examination was carried out for the enrolled patients after obtaining informed consent from them and their parents; affected primary teeth were identified, and radiographs were taken. Patients and parents were educated and motivated regarding the treatment by SDF and its outcome by showing pre- and postoperative photographs (Fig. 1).

SDF (38\%) (e-SDF by Kids-e-Dental; USA) was applied onto the carious lesion using an applicator tip and was allowed to absorb for 1 minute following proper isolation using cotton rolls and achieve dryness of the tooth which was a critical element of the procedure to maximize the ability of the SDF to seize the carious lesion. The surrounding area was isolated by applying petroleum jelly to avoid staining. The parents were made to watch the procedure and were educated that their child should not eat/drink for a minimum of 1 hour; further, the parents were asked to interact with their child regarding the procedure and their experience which would help them in filling the evaluation pro forma in their next visit. Follow-up visit was scheduled after 1 week and 3 months.

The questionnaire was drafted with a 4-item 5-point Likerttype scale confining from strongly agree, agree, neutral, disagree to strongly disagree. It was prepared concerning the ease of procedure, pain, discoloration of teeth, and taste acceptance. ${ }^{5}$

In the first follow-up visit, Likert 4-item 5-level scale pro forma was made available to parents to express their perception about SDF application (Table 1). ${ }^{5}$ This was followed by a reevaluation of the lesion and application of SDF if needed. The next recall visit was made after 3 months to evaluate the treated lesion using ICDAS tactile criteria. The presence of yellow soft lesions, pain, sinus, or swelling was considered to indicate treatment failure (Figs 2 and 3).

\section{Results}

Thirty-six children were selected for the study. Clinical examination was done regarding active carious lesions. Parents of patients were informed regarding the treatment plan and subsequent observation follow-up. Out of 36 , two parents refused for the treatment; so, 34 children with 123 active carious lesions were registered in the present study. Out of these 34 children, 4 (with 17 carious lesion-14 anterior and 4 posteriors) were unsuccessful in turning up for recall visits. Thirty children (with 106 active carious lesion-71 anterior and 35 posterior) completed the study, and their parents submitted the duly filled "parent perception" evaluation pro forma.

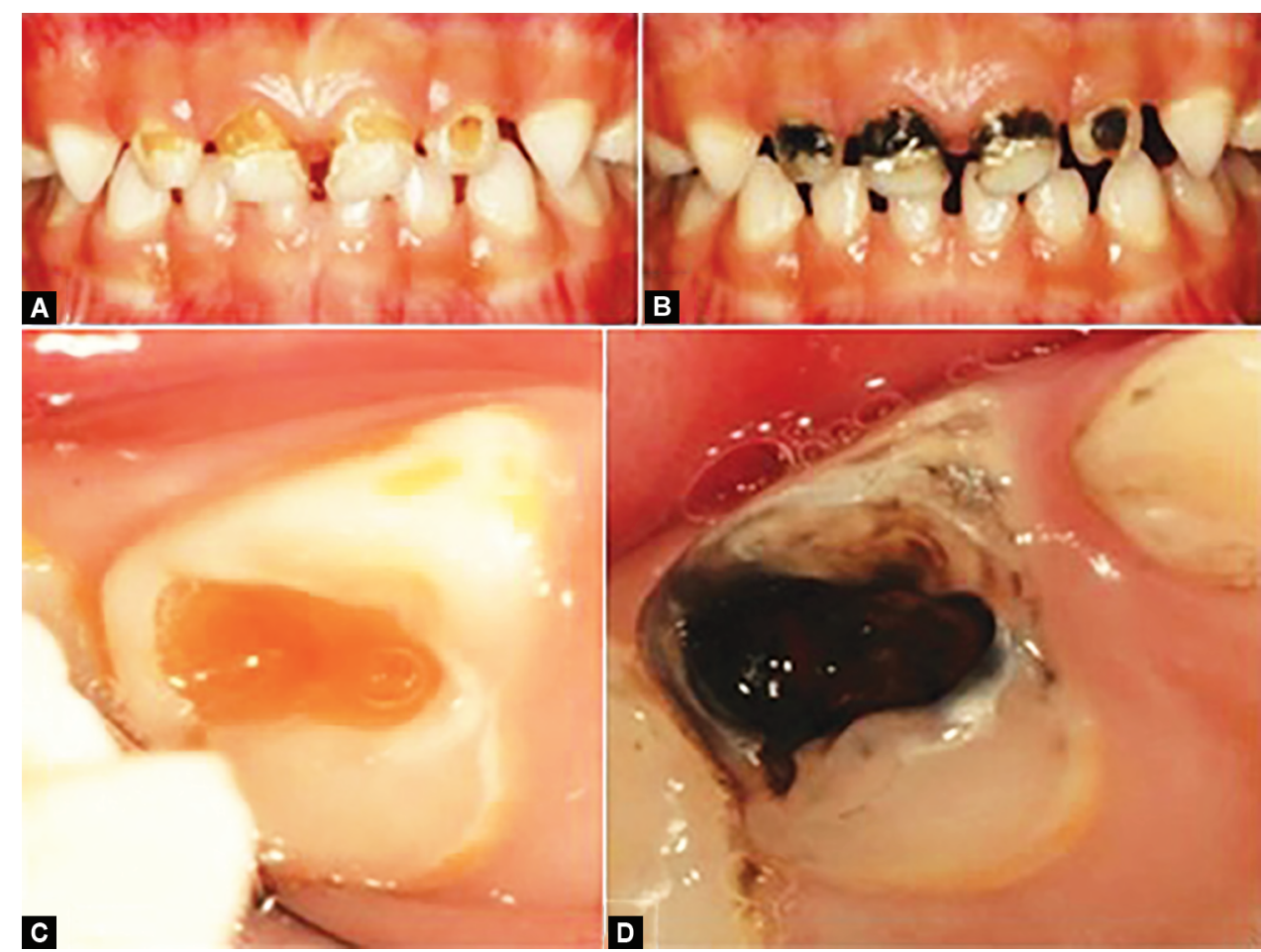

Figs 1 A to D: Pre- and postoperative photographs shown to the patients and parents before starting treatment (adopted from Alshammari et al. ${ }^{7}$ ) 
Table 1: Evaluation pro forma [4-item 5-point Likert scale]

\begin{tabular}{|c|c|c|c|c|c|c|}
\hline S. No. & Assertion & Strongly agree & Agree & Neutral & Disagree & $\begin{array}{l}\text { Strongly disa- } \\
\text { gree }\end{array}$ \\
\hline 1 & SDF application was an easy process & & & & & \\
\hline 2 & SDF application was a painless procedure & & & & & \\
\hline 3 & $\begin{array}{l}\text { Discoloration of cavities is acceptable as it } \\
\text { arrests caries progression }\end{array}$ & & & & & \\
\hline 4 & $\begin{array}{l}\text { As metallic taste of SDF goes away } \\
\text { with time it is acceptable }\end{array}$ & & & & & \\
\hline
\end{tabular}

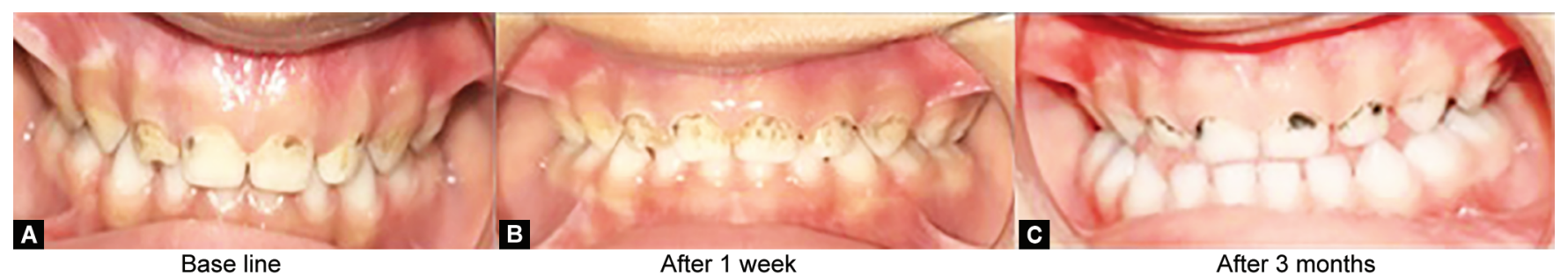

Figs 2 A to C: Preop and follow-up after application of SDF (patient X)

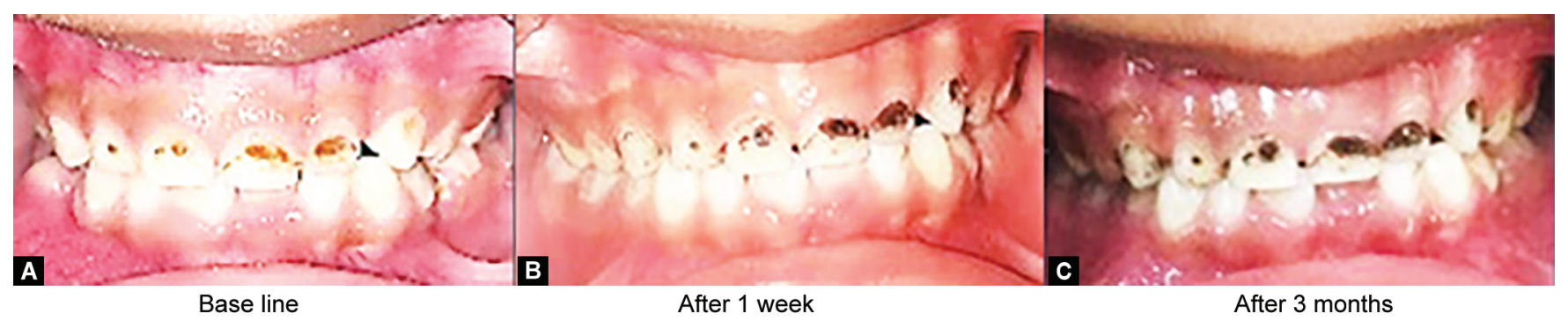

Figs 3 A to C: Preop and follow-up after application of SDF (patient $Y$ )

Table 2: Evaluation of carious lesion at different follow-up of the study period

\begin{tabular}{llcl}
\hline & \multicolumn{2}{c}{ Carious lesion $(n=30)$} & \\
\cline { 2 - 3 } Follow-up visits & Active, $n(\%)$ & Inactive, $n(\%)$ & p value \\
\hline After 1 week & $6(20.0)$ & $24(80)$ & $0.002(\mathrm{~S})$ \\
After 3 months & $2(6.7)$ & $28(93.3)$ & \\
\hline
\end{tabular}

\section{S, Significant}

The data collected subjected to statistical analysis using Statistical Package for Social Sciences (SPSS, IBM version 20.0) after it was entered in Microsoft Excel. The level of significance was considered statistically significant, as it was fixed at $5 \%$ and $p$ value $<0.05$. A Chi-square test was performed for quantitative variables.

The number of the active and inactive carious lesion after 1 week of application of SDF was found to be $20 \%$ and $80 \%$, respectively, whereas the same was recorded to be $6.7 \%$ and $93.3 \%$, respectively, after 3 months, which revealed a significant difference ( $p$ value $=0.002$ ) in efficacy between two follow-up visits with a greater number of inactive lesions after 3 months follow-up when compared to 1 week (Table 2 and Fig. 4).

Analysis of parent perception pro forma revealed that the majority of the parents agreed (49.2\%) or strongly agreed (46.7\%) that SDF application to be an easy process, a painless procedure with acceptable discoloration and taste while $4.1 \%$ had a neutral response. None of the parents disagreed or strongly disagreed in their opinion (Table 3 and Fig. 5).

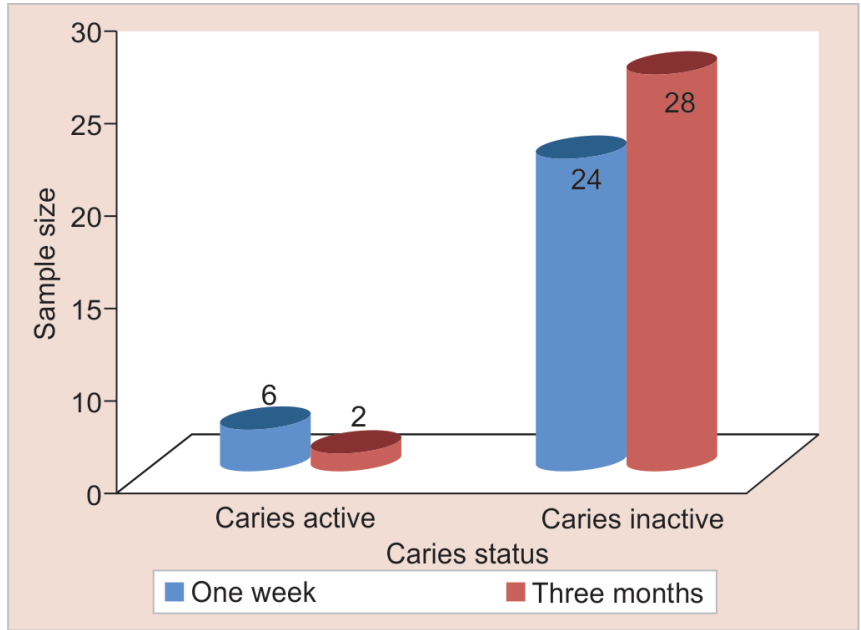

Fig. 4: Evaluation of carious lesion at different follow-up of the study period

\section{Discussion}

There is little literature concerning SDF effectiveness and parental perception. Our study is the first to contemplate on SDF parental perception in the central Indian population. The present short-term clinical study with limited samples demonstrates the usefulness of SDF in arresting active caries lesions in the deciduous dentition of children aged $2-10$ years and suggested that SDF is a suitable treatment option. 
Table 3: Analysis of parent perception evaluation pro forma

\begin{tabular}{|c|c|c|c|c|c|}
\hline Assertion & Strongly agree, $n(\%)$ & Agree, $n(\%)$ & Neutral, $n(\%)$ & Disagree, $n(\%)$ & Strongly disagree, $n(\%)$ \\
\hline SDF application was an easy process & $24(80)$ & $4(13.3)$ & $2(6.7)$ & - & - \\
\hline $\begin{array}{l}\text { SDF application was a painless } \\
\text { procedure }\end{array}$ & $18(60)$ & $11(36.7)$ & $1(3.3)$ & - & - \\
\hline $\begin{array}{l}\text { Discoloration of cavities is acceptable } \\
\text { as it arrests caries progression }\end{array}$ & $5(16.7)$ & $23(76.7)$ & $2(6.7)$ & - & - \\
\hline $\begin{array}{l}\text { As metallic taste of SDF goes away } \\
\text { with time it is acceptable }\end{array}$ & $9(30)$ & $21(70)$ & - & - & - \\
\hline Total & $56(46.7)$ & $59(49.2)$ & $5(4.1)$ & - & - \\
\hline
\end{tabular}

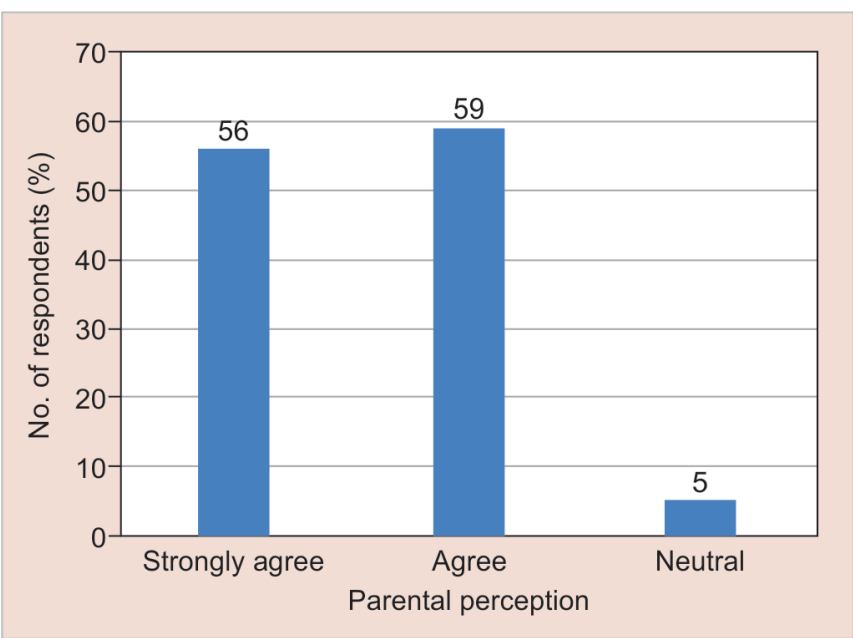

Fig. 5: Analysis of parent perception evaluation pro forma

In the present study, we have used SDF $38 \%$ concentration, as it is found to bring about significant greater inhibition of Matrix Metallo-Proteinases (MMP) than $30 \%$ and $12 \%$ SDF. $^{8}$

It has been mentioned that the yearly application of SDF is an effective remedy to apprehend active carious lesions of the dentinal region. ${ }^{9,10}$ But Zhi et al. and Horst et al. observed that upon increasing the application frequency from annual to every 6 months, the section of active caries arrest was augmented. ${ }^{11,12}$ Results of this study are in constant with the conclusion by Clemens et al. ${ }^{5}$ that the effectiveness of SDF can be enhanced by escalating the frequency of application from yearly to every 6 months.

Typically, patients with high carious activity are encouraged for frequent recall; thus, the bi-annual application will also coincide with recall and follow-up for such young children, and thus improved effects are expected with use of SDF. ${ }^{13}$

Results from a cross-sectional study by Bagher et al. ${ }^{14}$ supported the common parental notion regarding the use of SDF. They comprehended that more parents are comfortable with the staining connected with SDF treatment on their child's deciduous teeth when compared to permanent teeth, and also on posterior teeth, as a contrast to anterior teeth because of esthetic concerns. In our study, parental acceptance for SDF application in primary teeth was above $95 \%$ ( 106 active carious lesions-71 anterior and 35 posterior).

A study by Chu et al. ${ }^{15}$ reported that the application of SDF (38\%) arrested the carious lesions up to $84 \%$, which was found to be $93 \%$ in our study. Yee et al. found that only a single application of $38 \%$ SDF was effective in arresting caries after 2 years, and they also concluded that SDF $38 \%$ was more effective when compared to $12 \% .{ }^{16}$ Thus, the finding of our study is consistent with previous studies $8,15,16$ in terms of caries activity inhibition with every application. Our study results are compatible with the observation by Llodra et al., ${ }^{17} 3$ years controlled clinical trial, and they concluded that SDF in the concentration of $38 \%$ solution is effectual to control caries in deciduous teeth. Whereas Hihara et al. ${ }^{18}$ found a reduction in caries activity following the application of SDF at a concentration of $52 \%$ while the reduction in new caries development by SDF $42 \%$.

In a study by Crystal et al., ${ }^{19}$ parental acceptance was $75 \%$, which was $83 \%$ in our study. Clemens et al. ${ }^{5}$ measured parental acceptability by using a 4-item, 5 -level Likert-type scale and found it to be $83 \%$ with the same being $91 \%$ in our study.

\section{Conclusion}

Our results emphasize that for deciduous dentition, SDF is capable of preventing active dental decay lesions and also confirm the superb parental notion. SDF put forward an uncomplicated, fairly proficient, and well-conventional noninvasive remedy for dental decay in young children.

\section{Clinical Significance}

SDF is a noninvasive modality that could be chosen by parents looking for treatment without drills and fills, as these may cause any emotional and psychological influences onto the children. SDF may be a boon for uncooperative pediatric patients and also children with special healthcare need to stop active caries. Although it has a drawback of discoloration of teeth and metallic taste, parents show a positive attitude toward this treatment option.

\section{ACKNOWLedgments}

The authors are very grateful to the parents and children for their kind support and participation in the present study. Also, the investigators show gratitude to all the supporting employees of the Department of Pediatric and Preventive Dentistry, SAIMS, Indore for rendering support.

\section{References}

1. Twetman S. Prevention of dental caries as a non-communicable disease. Eur J Oral Sci 2018;126(S1):19-25. DOI: 10.1111/eos.12528

2. Moynihan P, Petersen PE. Diet, nutrition, and the prevention of dental diseases. Public Health Nutr 2004;7(1a):201-226. DOI: 10.1079/PHN2003589

3. Shah S, Bhaskar V, Venkatraghavan $\mathrm{K}$, et al. Silver diamine fluoride: a review and current applications. J Adv Oral Res 2014;5(1):25-35. DOI: $10.1177 / 2229411220140106$ 
4. Shounia TY, Atwan S, Alabduljabbar R. Using silver diamine fluoride to arrest dental caries: a new approach in the US. J Dent Oral Biol 2017;2(18):1105.

5. Clemens J, Gold J, Chaffin J. Effect and acceptance of silver diamine fluoride treatment on dental caries in primary teeth. J Pub Health Dent 2018;78(1):63-68. DOI: 10.1111/jphd.12241

6. Dikmen B. ICDAS II criteria (international caries detection and assessment system). J Istanbul Uni Facul Dent 2015;49(3):63-72. DOI: 10.17096/jiufd.38691

7. Alshammari AF, Almuqrin AA, Aldakhil AM, et al. Parental perceptions and acceptance of silver diamine fluoride treatment in the Kingdom of Saudi Arabia. Int J Health Sci 2019;13(2):25.

8. Mei ML, Ito L, Cao Y, et al. Inhibitory effect of silver diamine fluoride on dentine demineralization and collagen degradation. J Dent 2013;41(9):809-817. DOI: 10.1016/j.jdent.2013.06.009

9. $\mathrm{Chu} \mathrm{CH}$, Lo EC. Promoting caries arrest in children with silver diamine fluoride: a review. Oral Health Prevent Dent 2008;6(4):315-321.

10. Rosenblatt A, Stamford TC, Niederman R. Silver diamine fluoride: caries "silver-fluoride bullet". J Dent Res2009;88(2):116-125. DOI: 10.1177/0022034508329406

11. Zhi QH, Lo EC, Lin HC. Randomized clinical trial on the effectiveness of silver diamine fluoride and glass ionomer in arresting dentine caries in preschool children. J Dent 2012;40(11):962-967. DOI: 10.1016/ j.jdent.2012.08.002

12. Horst JA, Ellenikiotis $H$, Milgrom PM. UCSF silver caries arrest committee. UCSF protocol for caries arrest using silver diamine fluoride: rationale, indications, and consent. J Califor Dent Associat 2016;44(1):16.

13. Sharma G, Puranik MP. Approaches to arresting dental caries: an update J Clin Diagnos Res: JCDR 2015;9(5):ZE08. DOI: 10.7860/ JCDR/2015/12774.5943

14. Bagher SM, Sabbagh HJ, AlJohani SM, et al. Parental acceptance of the utilization of silver diamine fluoride on their child's primary and permanent teeth. Patient Prefer Adher 2019;13:829. DOI: 10.2147/PPA. S205686

15. Chu CH, Lo EC, Lin HC. Effectiveness of silver diamine fluoride and sodium fluoride varnish in arresting dentin caries in Chinese preschool children. J Dent Res 2002;81(11):767-770. DOI: 10.1177/0810767

16. Yee R, Holmgren C, Mulder J, et al. Efficacy of silver diamine fluoride for arresting caries treatment. J Dent Res2009;88(7):644-647. DOI: $10.1177 / 0022034509338671$

17. Llodra JC, Rodriguez A, Ferrer B, et al. Efficacy of silver diamine fluoride for caries reduction in primary teeth and first permanent molars of schoolchildren: A 36-month clinical trial. J Dent Res2005;84(8): 721-724. DOI: 10.1177/154405910508400807

18. Hihara T, Nishino M, Yasutomi Y, et al. Effects of diamine silver fluoride on arrestment and prevention of caries in primary teeth. Dent Jpn (Tokyo) 1994;31:93-95.

19. Crystal YO, Janal MN, Hamilton DS, et al. Parental perceptions and acceptance of silver diamine fluoride staining. The Journal of the American Dental Association 2017;148(7):510-518. DOI: 10.1016/ j.adaj.2017.03.013 